\title{
Thin Film Cracking Modulated by Underlayer Creep
}

\author{
by J. Liang, R. Huang, J.H. Prévost, and Z. Suo
}

\begin{abstract}
In devices that integrate dissimilar materials in small dimensions, crack extension in one material often accompanies inelastic deformation in another. In this paper we analyze a channel crack advancing in an elastic film, while an underlayer creeps. The film is subject to a tensile stress. As the underlayer creeps, the stress field in the film relaxes in the crack wake, and intensifies around the crack tip. In a blanket film, the crack can attain a steady velocity, set by two rate processes: subcritical decohesion at the crack tip, and creep in the underlayer. In a thin-film microbridge over a viscous stripe, the crack cannot grow when the bridge is short, and can grow at a steady velocity when the bridge is long. We use a two-dimensional shear lag model to approximate the threedimensional fracture process, and an extended finite element method to simulate the moving crack with an invariant, relatively coarse mesh. On the basis of the theoretical findings, we propose new experiments to measure fracture toughness and creep laws in small structures. As a byproduct, an analytical formula is found for the growth rate per temperature cycle of a channel crack in a brittle film, induced by ratcheting plastic deformation in a metal underlayer.
\end{abstract}

KEY WORDS-Crack, thin film, creep, ratcheting

\section{Introduction}

Fracture in small structures has been studied intensely in recent years, motivated by diverse applications such as interconnects in microprocessors, resonant structures in microelectromechanical systems (MEMS), thermal barrier coatings in gas-turbine engines, and multilayers in medical implants. ${ }^{1-10}$ The applications typically require that materials with extremely different properties be integrated in small dimensions. The structural complexity, as well as the small feature sizes, can lead to unusual phenomena. For example, it has been discovered that cracks can grow in brittle films under cyclic temperatures, driven by ratcheting plastic deformation in a metal underlayer. ${ }^{11-15}$ We will revisit this phenomenon towards the end of this paper.

In the study of fracture in small dimensions, a prototype structure consists of a thin brittle film on a substrate.

\footnotetext{
J. Liang is a Graduate Student and Z. Suo (suo@deas.harvard.edu) is a Professor, Department of Mechanical and Aerospace Engineering and Princeton Materials Institute, Princeton, NJ 08544. R. Huang is a Professor and J.H. Prévost is a Professor, Department of Civil and Environmental Engineering, Princeton University, Princeton, NJ 08544. R. Huang is currently at Aerospace Engineering and Engineering Mechanics Department, University of Texas at Austin, Austin, TX 78712-1085.
}

Original manuscript submitted: December 19, 2002.

Final manuscript received: December 19, 2002.
The film is under a residual tensile stress, which may drive pre-existing flaws to grow into channel cracks in the film. The film-on-substrate differs from a free-standing sheet in an obvious way; the substrate constrains the film. ${ }^{4}$ For both elastic and plastic substrates, the stress intensity factor at the channel front depends on the film thickness, rather than the channel length. ${ }^{16-19}$ A critical film thickness exists, below which no pre-existing flaws can grow into channels, no matter how large these flaws are.

Brittle films on inelastic substrates are ubiquitous in practice. For instance, silicon dioxide films on polymer foils serve as oxygen-barrier packaging materials in the pharmaceutical and food industries. ${ }^{20}$ Aluminum oxide scales on alloys form environmental barriers at elevated temperatures. ${ }^{9,10}$ Silicon nitride films are used as passivation in microelectronic devices. ${ }^{11-13}$ Semiconductor thin films have been wafer bonded to viscous substrates to fabricate strain-relaxed, crack-free islands. ${ }^{21,22}$ Because metals, and more recently organic materials,${ }^{23-25}$ are pervasive in electronic and photonic devices, it is urgent to study time-dependent deformation in small structures.

Figure 1 illustrates the structures to be studied in this paper. A blanket film, thickness $h$, lies on an underlayer, thickness $H$, which in turn lies on a substrate; see Fig. 1(a). The film is elastic, the underlayer viscous, and the substrate rigid. They are well bonded. Initially, the film is in a uniform biaxial tensile stress state; the in-plane misfit strain is $\varepsilon_{0}$. When the underlayer creeps, the stress field in the film relaxes in the crack wake, but intensifies around the crack tip. We have studied the stationary crack previously, ${ }^{26}$ and we study the moving crack in this paper. When the crack tip moves slowly, the crack wake has a long time to relax, and the stress intensity around the crack tip increases. When the crack tip moves rapidly, the crack wake has a short time to relax, and the stress intensity around the crack tip decreases. Consequently, the crack can attain a steady velocity. Underlayer creep modulates thin-film cracking.

We also consider an elastic microbridge, length $2 L$, over a viscous stripe. Figures 1(b) and 1(c) illustrate two structures commonly used in interconnects. We assume that $L \gg h$ and $L \gg H$, and the two ends of each bridge are rigidly held by the substrate. The crack breaks the bridge in the middle, and is in the mode I condition. So long as the crack behavior is concerned, the two structures in Figs. 1(b) and 1(c) are equivalent. First, we assume that the crack tip is stationary. After some time, the bridge reaches the equilibrium state, and the underlayer carries no stress and stops creeping. In the equilibrium state, the stress intensity factor depends on the bridge length $2 L$, rather than its thickness $h$. For the crack tip to be stationary, this equilibrium stress intensity factor must be 


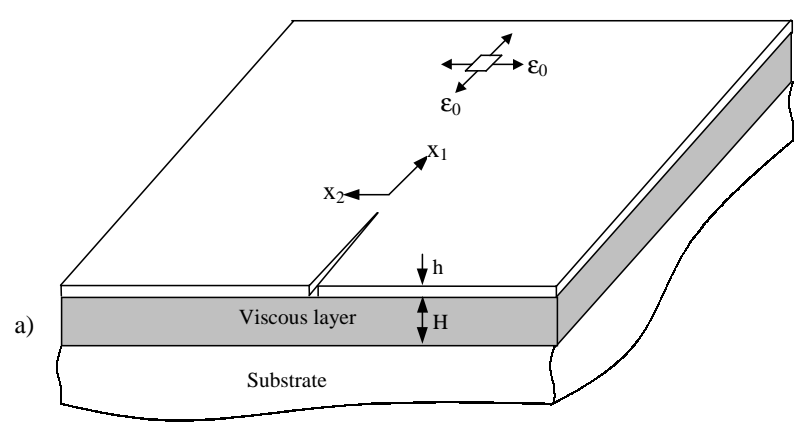

b)

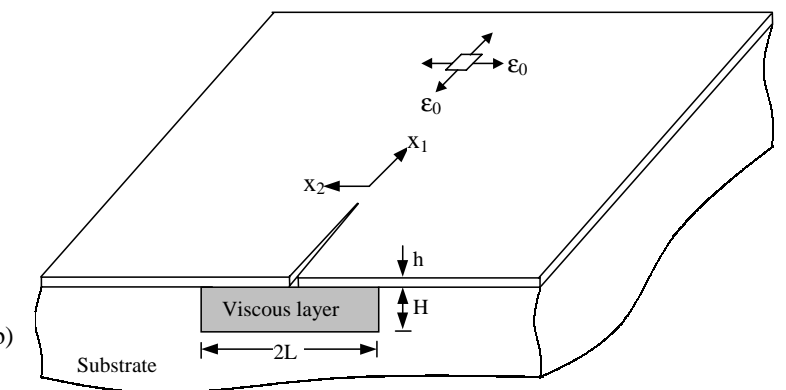

c)

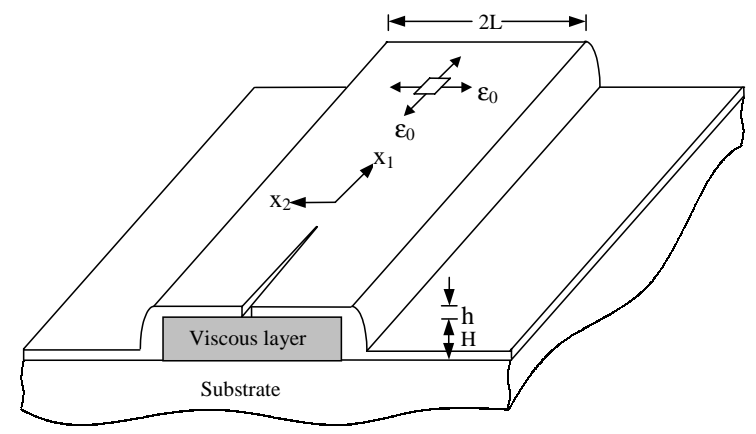

Fig. 1-(a) A blanket elastic film lies on a viscous underlayer. The film is under a biaxial residual stress, motivating a channel crack to grow in the film. (b), (c) An elastic microbridge is over a viscous stripe, with the two ends constrained by the substrate. The length of the bridge is much larger than the thicknesses of the layers.

below the toughness. Consequently, a critical bridge length exists, below which the crack tip will never move. Above this critical length, the crack tip will move and attain a steady velocity.

We show that both the steady crack velocity and the equilibrium stress intensity factor are robust quantities, which can be realized under a wide range of conditions. On the basis of these findings, we propose novel methods to measure fracture toughness and creep laws. We also show how to extend this study for substrate undergoing ratcheting deformation or nonlinear creep.

\section{The Model}

\section{The Two-Dimensional Shear Lag Model}

Recently, a one-dimensional shear lag model has been formulated to study strain relaxation in an elastic island on a viscous layer. ${ }^{27,28}$ The model has been extended to two dimensions, ${ }^{26,29}$ and is summarized here. The surface of the film coincides with the coordinate plane $\left(x_{1}, x_{2}\right)$. We take the film under the uniform biaxial stress as the reference state, in which the displacement field vanishes. At time $t$, relative to the reference state, the in-plane displacement field is $u_{\alpha}\left(x_{1}, x_{2}, t\right)$. The Greek subscript takes values 1 and 2 . The in-plane strain field in the film, $\varepsilon_{\alpha \beta}\left(x_{1}, x_{2}, t\right)$, is the sum of that due to the misfit strain, and that due to the displacement gradient, namely

$$
\varepsilon_{\alpha \beta}=\varepsilon_{0} \delta_{\alpha \beta}+\frac{1}{2}\left(\frac{\partial u_{\alpha}}{\partial x_{\beta}}+\frac{\partial u_{\beta}}{\partial x_{\alpha}}\right),
$$

where $\delta_{\alpha \beta}=1$ when $\alpha=\beta$, and $\delta_{\alpha \beta}=0$ when $\alpha \neq \beta$.

The crack velocity is typically much smaller than the elastic wave speed, so that the inertia effect is negligible. The membrane stresses $\sigma_{\alpha \beta}$ act in the film, and the underlayer exerts shear stresses $\tau_{\alpha}$ on the bottom face of the film. The force balance of a differential element of the film requires that

$$
\frac{\partial \sigma_{\alpha \beta}}{\partial x_{\beta}}=\frac{\tau_{\alpha}}{h} .
$$

We adopt the convention that a repeated Greek subscript implies summation over 1 and 2. Because the film is thin, the shear stresses acting on the bottom face of the film are equivalent to body forces, of magnitude $-\tau_{\alpha} / h$ per unit volume, distributed throughout the film. In effect, the film is in a state of plane stress subject to the body forces. We can also obtain eq (2) by integrating the three-dimensional force balance equations through the film thickness, interpreting $\sigma_{\alpha \beta}$ as the average stress over the film thickness.

The film is elastic and isotropic. The membrane stresses relate to the in-plane strains by Hooke's law

$$
\sigma_{\alpha \beta}=\frac{E}{1-\nu^{2}}\left[(1-\nu) \varepsilon_{\alpha \beta}+\nu \varepsilon \gamma \gamma \delta_{\alpha \beta}\right]
$$

where $E$ is Young's modulus, and $\nu$ is Poisson's ratio.

The underlayer is also thin, so that the shear stresses $\tau_{\alpha}$ are taken to be uniform across the thickness of the underlayer. For the time being, we assume that the underlayer is linear viscous, with the viscosity $\eta$. The shear strain rates, $\left(\partial u_{\alpha} / \partial t\right) / H$, relate to the shear stresses $\tau_{\alpha}$ as

$$
\frac{1}{H} \frac{\partial u_{\alpha}}{\partial t}=\frac{\tau_{\alpha}}{\eta} .
$$

Equations (1)-(3) define a plane stress problem with body forces. Equation (4) evolves the body forces. A combination of eqs (1)-(4) gives

$$
\frac{\partial u_{\alpha}}{\partial t}=D\left[\frac{1}{2(1+\nu)} \frac{\partial^{2} u_{\alpha}}{\partial x_{\beta} \partial x_{\beta}}+\frac{1}{2(1-\nu)} \frac{\partial^{2} u_{\beta}}{\partial x_{\beta} \partial x_{\alpha}}\right],
$$

where

$$
D=h H E / \eta \text {. }
$$


Equation (5), which evolves the displacement field $u_{\alpha}\left(x_{1}, x_{2}, t\right)$, is analogous to the diffusion equation, with $D$ as the effective diffusivity. As time passes, the relaxation starts at the crack, and diffuses into the interior of the film.

\section{Singular Field at the Tip of a Moving Crack}

For a stationary crack, the displacement field is bounded at the crack tip and smooth in time, save for the sudden start of the relaxation process. Consequently, according to eq (4), the shear stresses $\tau_{\alpha}$ are bounded at the crack tip. When the crack tip moves, however, the shear stresses $\tau_{\alpha}$ are singular at the crack tip. Following an established method, ${ }^{30,31}$ we next show that the singular field at the tip of a moving crack is still identical to that of a stationary crack under the plane stress conditions.

In general, the crack is a curve in the plane $\left(x_{1}, x_{2}\right)$. The coordinates of the crack tip are functions of time, $x_{1}=a_{1}(t)$ and $x_{2}=a_{2}(t)$. The crack velocity $\mathrm{d} a_{\alpha} / \mathrm{d} t$ can be timedependent. Let $\left(\bar{x}_{1}, \bar{x}_{2}\right)$ be the moving frame whose origin coincides with the crack tip. A material particle with coordinates $\left(x_{1}, x_{2}\right)$ in the stationary frame has the coordinates

$$
\bar{x}_{\alpha}=x_{\alpha}-a_{\alpha}(t)
$$

in the moving frame.

Writing eq (5) in the moving frame for the displacement field $u_{\alpha}\left(\bar{x}_{1}, \bar{x}_{2}, t\right)$, we obtain that

$$
\begin{aligned}
& -\frac{\partial u_{\alpha}}{\partial \bar{x}_{\beta}} \frac{d a_{\beta}}{d t}+\frac{\partial u_{\alpha}}{\partial t} \\
& =D\left[\frac{1}{2(1+\nu)} \frac{\partial^{2} u_{\alpha}}{\partial \bar{x}_{\beta} \partial \bar{x}_{\beta}}+\frac{1}{2(1-\nu)} \frac{\partial^{2} u_{\beta}}{\partial \bar{x}_{\beta} \partial \bar{x}_{\alpha}}\right] .
\end{aligned}
$$

At the crack tip, the stress field is singular in $\bar{x}_{1}$ and $\bar{x}_{2}$, but smooth in $t$. The terms $\partial u_{\alpha}\left(\bar{x}_{1}, \bar{x}_{2}, t\right) / \partial t$ are bounded, but the terms $\partial u_{\alpha}\left(\bar{x}_{1}, \bar{x}_{2}, t\right) / \partial \bar{x}_{\beta}$ are singular, so that the shear stresses $\tau_{\alpha}$ are indeed singular. However, the terms $\partial u_{\alpha}\left(\bar{x}_{1}, \bar{x}_{2}, t\right) / \partial \bar{x}_{\beta}$ are one order less singular than the terms on right-hand side of eq (8), so that the singular field is determined by dropping the left-hand side in eq (8), and setting the quantity in the bracket to zero. The latter is identical to the governing equation for the elastic plane stress field with no body forces. Consequently, the singular crack tip field for the moving crack is identical to that for the stationary crack. This conclusion differs from that for a crack moving at a high velocity when the inertia effect is important. In that case, the acceleration of material particles (i.e., the second time derivative of the displacement) enters, and the singular stress field depends on the crack velocity. ${ }^{30,31}$

In this paper, we study the crack moving along the $x_{1}$-axis, under the mode I condition. The stress, a distance $\bar{x}_{1}$ ahead of the crack, takes the conventional form

$$
\sigma_{22}\left(x_{1}, t\right)=\frac{K(t)}{\sqrt{2 \pi \bar{x}_{1}}},
$$

where $K$ is the stress intensity factor, which depends on time, as indicated.

Let $K_{c}$ be the toughness of the film. For the time being, we neglect subcritical decohesion, and assume that the crack tip is stationary when $K<K_{c}$, and moves when $K=K_{c}$.
This crack growth criterion, together with the field equation (5) and the boundary conditions, sets up a moving boundaryvalue problem, which simultaneously evolves the displacement field $u_{\alpha}\left(x_{1}, x_{2}, t\right)$ and the crack tip location $a_{1}(t)$.

\section{Force Balance, Equilibrium State, and Steady State}

The force balance, eq (2), which is maintained at all time, does not guarantee thermodynamic equilibrium. In a thermodynamic equilibrium state, the underlayer stops creeping, the shear stresses $\tau_{\alpha}$ vanish, and the crack tip stops moving. The governing equation (5) becomes

$$
\frac{1}{2(1+\nu)} \frac{\partial^{2} u_{\alpha}}{\partial x_{\beta} \partial x_{\beta}}+\frac{1}{2(1-\nu)} \frac{\partial^{2} u_{\beta}}{\partial x_{\beta} \partial x_{\alpha}}=0 .
$$

In equilibrium, the film is under the plane stress conditions, with no body forces. For example, consider a square film island, subject to a biaxial stress initially, and free to relax from the edges. When the island reaches the equilibrium state, the stress in the island vanishes. As a second example, consider an island with the edges rigidly held by the substrate. The island under the initial stress is in an equilibrium state. A third example concerns the microbridge in Figs. 1(b) or 1(c). When the underlayer stops creeping, the bridge is no longer affected by the underlayer, but is still held at the two ends by the substrate. The plane stress field in the film is non-uniform, which will be studied in a later section.

As discussed in the introduction, we also encounter the steady state, in which the crack tip moves at a constant velocity, and the field in the film is time-independent to an observer moving at the same velocity as the crack tip. In the steady state, the material particles in the film still move, and the shear stresses in the underlayer do not vanish. Consider a crack moving in the $x_{1}$-direction at a steady velocity $V_{s s}$. The moving frame relates to the stationary frame as $\bar{x}_{1}=x_{1}-V_{s s} t$ and $\bar{x}_{2}=x_{2}$. In the moving frame, the displacement field is time-independent, $u_{\alpha}\left(\bar{x}_{1}, \bar{x}_{2}\right)$. The governing equation (5) becomes

$$
-V_{s s} \frac{\partial u_{\alpha}}{\partial \bar{x}_{1}}=D\left[\frac{1}{2(1+\nu)} \frac{\partial^{2} u_{\alpha}}{\partial \bar{x}_{\beta} \partial \bar{x}_{\beta}}+\frac{1}{2(1-\nu)} \frac{\partial^{2} u_{\beta}}{\partial \bar{x}_{\beta} \partial \bar{x}_{\alpha}}\right] .
$$

This equation, together with the boundary conditions and the crack growth criterion $K=K_{c}$, determines the steady-state displacement field and the steady crack velocity $V_{s s}$. In this paper, we do not use eq (11) to determine the steady state. Rather, we use a finite element method to evolve the field from the initial state to the steady state.

\section{Scales of Stress, Length and Time}

Before creep starts, the film is in a uniform biaxial stress state. The magnitude of the stress $\sigma_{0}$ relates to the misfit strain $\varepsilon_{0}$ as

$$
\sigma_{0}=\frac{E \varepsilon_{0}}{1-v}
$$

The stress $\sigma_{0}$ is the load in this problem, and scales the stress field at all time. In experiments, the residual stress can be measured by the wafer curvature method, and the misfit strain by X-ray diffraction. ${ }^{32,33}$ 
The thicknesses of the film and the underlayer, $h$ and $H$, enter the boundary-value problem through the effective diffusivity $D$. Consequently, $h$ and $H$ do not set the length scale of the problem. The problem, however, does have an intrinsic length:

$$
\Lambda=\left(K_{c} / \sigma_{0}\right)^{2} .
$$

For a moving crack, the length $\Lambda$ scales the zone over which the stress field varies rapidly. Far away from the crack, the film is in the state of biaxial stress $\sigma_{0}$. The shear lag model applies when the two thicknesses, $h$ and $H$, are small compared to $\Lambda$. Using the representative values, $K_{c}=1 \mathrm{MPa} \sqrt{\mathrm{m}}$ and $\sigma_{0}=$ $500 \mathrm{MPa}$, we obtain the intrinsic length $\Lambda=4 \mu \mathrm{m}$.

The diffusion-like eq (5) also has an intrinsic time

$$
t_{0}=\frac{\Lambda^{2}}{D}=\frac{K_{c}^{4} \eta}{\sigma_{0}^{4} H h E} .
$$

This time allows events over the length scale $\Lambda$ to take place. The intrinsic time $t_{0}$ is proportional to $\eta / E$, which has the unit of time, and to the length ratio $\Lambda^{2} /(H h)$. Using the representative values, $\Lambda=4 \mu \mathrm{m}, E=10^{11} \mathrm{~N} \mathrm{~m}^{-2}, \eta=$ $10^{10} \mathrm{sN} \mathrm{m}^{-2}, h=0.1 \mu \mathrm{m}$, and $H=1 \mu \mathrm{m}$, we obtain the intrinsic time $t_{0}=16 \mathrm{~s}$.

\section{Notes on Numerical Implementation}

The extended finite element method, ${ }^{34-37}$ developed by Belytschko and co-workers recently, is adapted for the present problem. The method simulates a moving crack with an invariant, relatively coarse mesh. We implement the method within DYNAFLOW, a general purpose finite element program developed by Prévost over the last two decades. ${ }^{38}$ In a previous paper, ${ }^{26}$ we have described a procedure to calculate the time-dependent stress intensity factor and displacement field. We now use the procedure to move the crack tip (Fig. 2). At a given time, we hold the crack tip location fixed, and calculate $K$ at the crack tip. If $K<K_{c}$, we hold the crack tip location fixed. If $K \geq K_{c}$, we instantaneously relocate the crack tip by a prescribed distance, $\delta a$. Because the crack tip now is in an unrelaxed film, $K$ drops below $K_{c}$. We hold the crack tip at the new location, evolve the displacement field, and record the time interval $\delta t$ for $K$ to climb back to $K_{c}$. We repeat the procedure to advance the crack over a long time. The crack velocity, $V$, is calculated from $V \approx \delta a / \delta t$.

In the simulation, the crack extension $\delta a$ is arbitrarily prescribed, but the time interval $\delta t$ is computed. In principle, smaller $\delta a$ yields higher accuracy; in practice, however, $\delta a$ has to be at least one element size. For simplicity, we use a uniform mesh in all calculations. Although the extended finite element method does not require a fine mesh to resolve the crack tip singularity, we do need to resolve the field over the dimension scaled by the intrinsic length $\Lambda$. The time step in updating the displacement should be small compared to $\delta t$. After trials, we find that the element size $0.02 \Lambda$ and the time step $0.0025 t_{0}$ are adequate. In all calculations we assume that Poisson's ratio is $\nu=0.3$.

\section{Results}

\section{Crack in a Blanket Film}

Let $S$ be a representative length of the lateral features, e.g., the bridge length or the initial crack length. The film

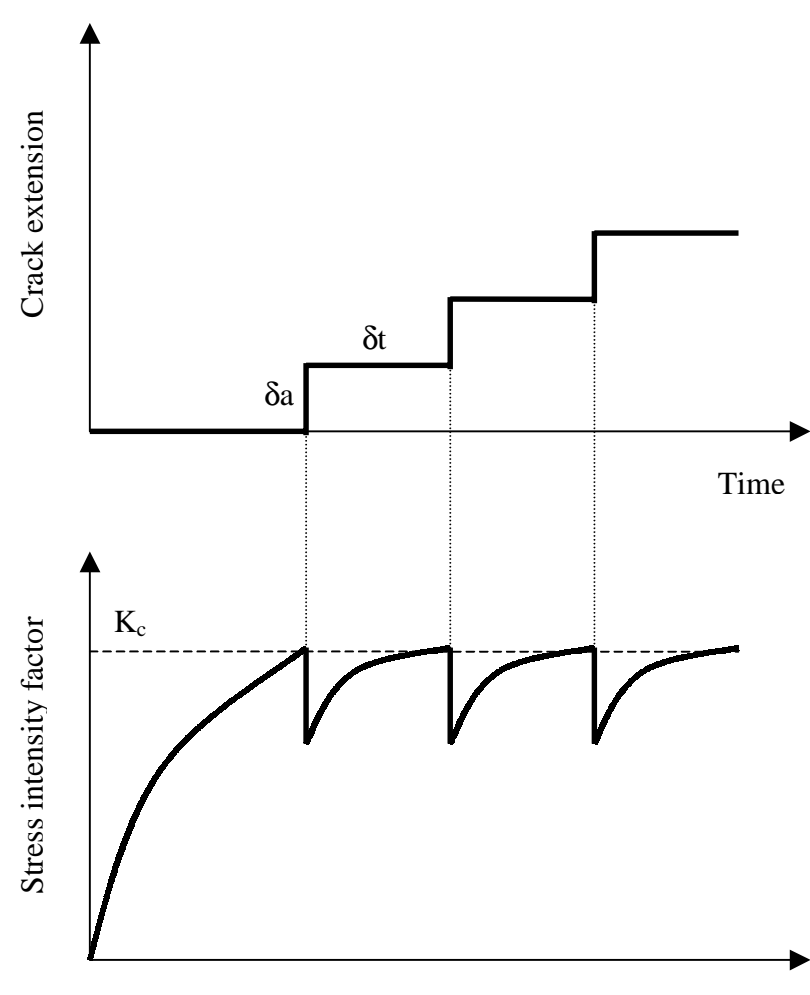

Time

Fig. 2-Schematic diagram of the numerical procedure to move the crack tip. (a) The crack extension as a function of time. (b) The stress intensity factor as a function of time.

may be regarded as infinite, and the crack semi-infinite, when $S \gg \Lambda$ and the time duration of interest is much shorter than $S^{2} / D$. In the simulation, we used a square film island, and prescribed on the four edges zero normal displacement and zero shear traction. After trials, we found that the square of side $10 \Lambda$ adequately represented an infinite film for a crack of initial length $5 \Lambda$ to extend about $2 \Lambda$. Figures 3-5 show the simulation results of this case.

Figure 3 gives the time sequence of the stress intensity factor $K$, the crack extension $a$, and the crack velocity $V$. We can partition the time sequence into three periods: stationary crack, transient moving crack, and steady moving crack. We have modeled the underlayer as a viscous material and neglected elasticity. Before creep begins, the underlayer is rigid, and the film has the uniform stress $\sigma_{0}$. Consequently, $K=0$ at $t=0$. After the underlayer starts to creep, the stress field in the film relaxes in the crack wake, and $K$ increases with time. Before $K$ attains $K_{c}$, the crack tip is stationary, and the length $\Lambda$ does not enter the problem. The problem lacks any fixed length scale, but has the diffusion length $\sqrt{D t}$. The stress intensifies around the crack tip in a zone on the scale $\sqrt{D t}$, and is $\sigma_{0}$ far away from the crack. Dimensional considerations dictate that $K$ should take the form

$$
K=\kappa \sigma_{0}(D t)^{1 / 4},
$$

where $\kappa$ is a dimensionless number depending only on Poisson's ratio and has been tabulated. ${ }^{26}$ We find $\kappa=1.07$ when $\nu=0.3$. The difference in $\kappa$ between the value reported here 

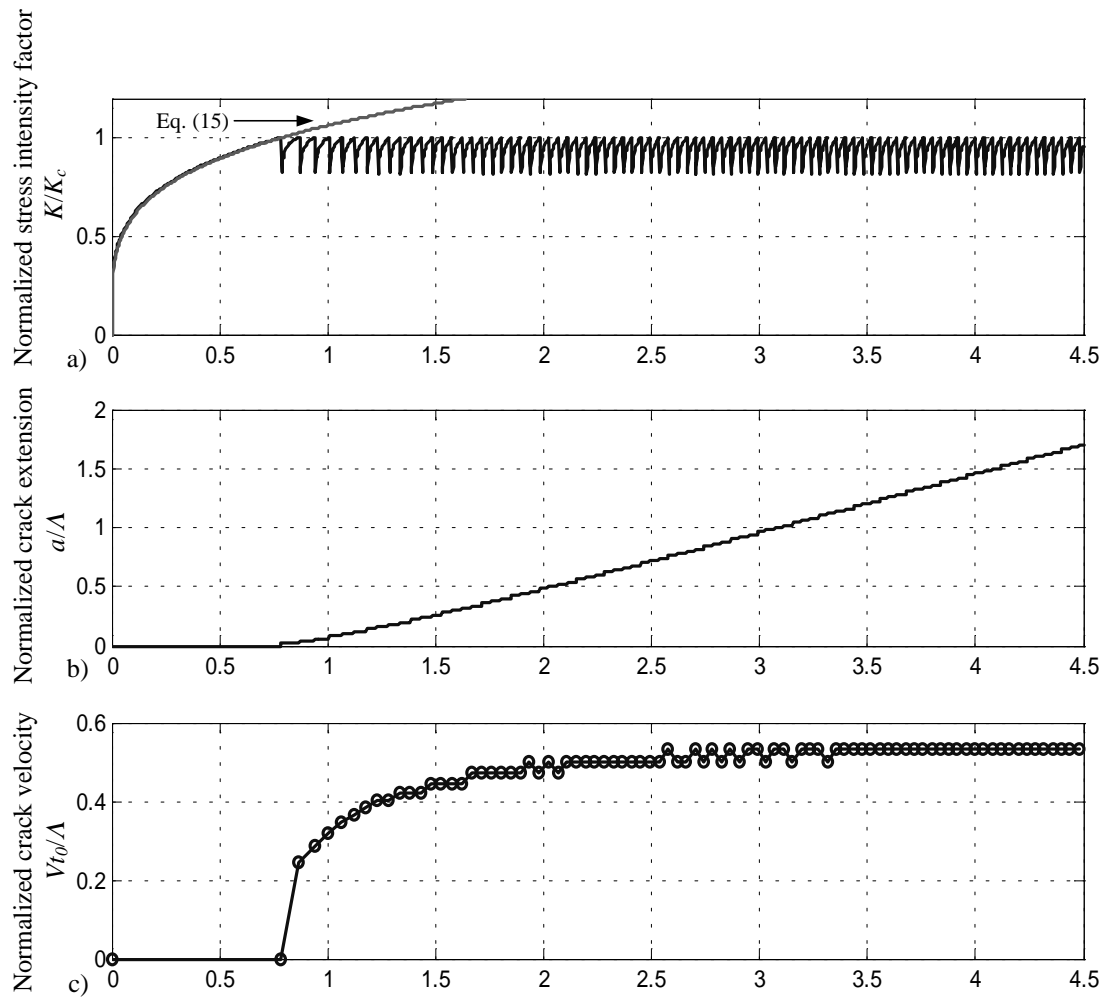

Fig. 3-Numerical results for a square film of side $10 \Lambda$ and initial crack length $5 \Lambda$. The normal displacements on all sides are held fixed. (a) The stress intensity factor as a function of time. (b) The crack extension as a function of time. (c) The crack velocity as a function of time.

and in Huang et al. ${ }^{26}$ is due to the different normalization used for $K$, a different definition of $D$, and a finer mesh and smaller time step used in the calculations in this paper. The numerical results in Fig. 3(a) reproduce the scaling law (15).

At $t=t_{0} / \kappa^{4}=0.763 t_{0}, K=K_{c}$, and the crack tip starts to move. The crack velocity increases with time, and attains a steady value after the tip moves a distance about $\Lambda$. Dimensional considerations require that the steady velocity for a crack in a blanket film, $V_{s s}^{\infty}$, obey the scaling law

$$
V_{s s}^{\infty}=\chi \frac{\Lambda}{t_{0}}=\chi \frac{H h E \sigma_{0}^{2}}{\eta K_{c}^{2}},
$$

where $\chi$ is a dimensionless number depending on Poisson's ratio. The numerical results in Fig. 3(c) give $\chi=0.534$ for $\nu=$ 0.3 . Using the representative values of $\Lambda$ and $t_{0}$ given before, the steady velocity is of the order of $0.1 \mu \mathrm{m} \mathrm{s}^{-1}$. Indeed, the crack velocity is much below the elastic wave speed, which exceeds $1 \mathrm{~km} \mathrm{~s}^{-1}$ for typical materials.

Figure 4 plots contours of the stress field $\sigma_{22}$ at several times, giving the stress in units of $\sigma_{0}$, and the coordinates $x_{1}$ and $x_{2}$ in units of $\Lambda$. At $t=0.5 t_{0}$, the stress field relaxes in the crack wake, and intensifies around the crack tip; the crack tip is stationary. At $t=t_{0}$, the crack tip moves at a velocity below the steady value. After $t=2 t_{0}$, according to Fig. 3(c), the crack tip moves at the steady velocity. Figure 4 shows that, between $t=2 t_{0}$ and $t=4 t_{0}$, the field is invariant only in a small zone around the crack tip.

Figure 5 plots the crack opening displacement $u_{2}\left(x_{1}, \pm 0, t\right)$ at several times. At some distance behind the crack tip, the crack opening displacement is independent of the position $x_{1}$, and follows the one-dimensional diffusion-like behavior. ${ }^{27,28}$ The crack tip is stationary in the beginning, and moves at the steady velocity after $t=2 t_{0}$. Once again, the steady state is local; only the displacement profile in a small zone around the crack tip is invariant with time.

The simulation clearly shows that the crack tip attains the steady velocity long before the entire film attains the steady field. This behavior originates from the diffusive character of the problem. For the crack tip to attain the steady velocity, only the stress field in a zone of size about $\Lambda$ needs to evolve from the initial to the steady state; the time needed scales as $\Lambda^{2} / D$. For the film to attain the steady field, the stress field in the entire film must evolve from the initial to the steady state; the time needed scales as $S^{2} / D$. Of course, to maintain a steady velocity, the relaxation front from boundaries other than the crack, if present, should be far from the crack tip. For example, for a film island with free edges, when the relaxation front from the edges reaches the crack tip, the stress intensity factor drops, and the crack arrests inside the island.

Now consider a crack of initial length $2 a_{0}$ in a blanket film. It is well known that, for a free-standing elastic sheet under stress $\sigma_{0}$ remote from the crack, the stress intensity factor at the crack tip is $\sigma_{0} \sqrt{\pi a_{0}}$. For a blanket film on a viscous underlayer, if $\sigma_{0} \sqrt{\pi a_{0}}<K_{c}$, as the underlayer creeps, $K$ at the crack tip builds up from zero to $\sigma_{0} \sqrt{\pi a_{0}}$, so that the crack tip never moves. ${ }^{26}$ If $\sigma_{0} \sqrt{\pi a_{0}}>K_{c}, K$ at the crack tip builds up to $K_{c}$ and, soon afterwards, the crack tip moves at the steady velocity. Consequently, the steady velocity $V_{s s}^{\infty}$ is 

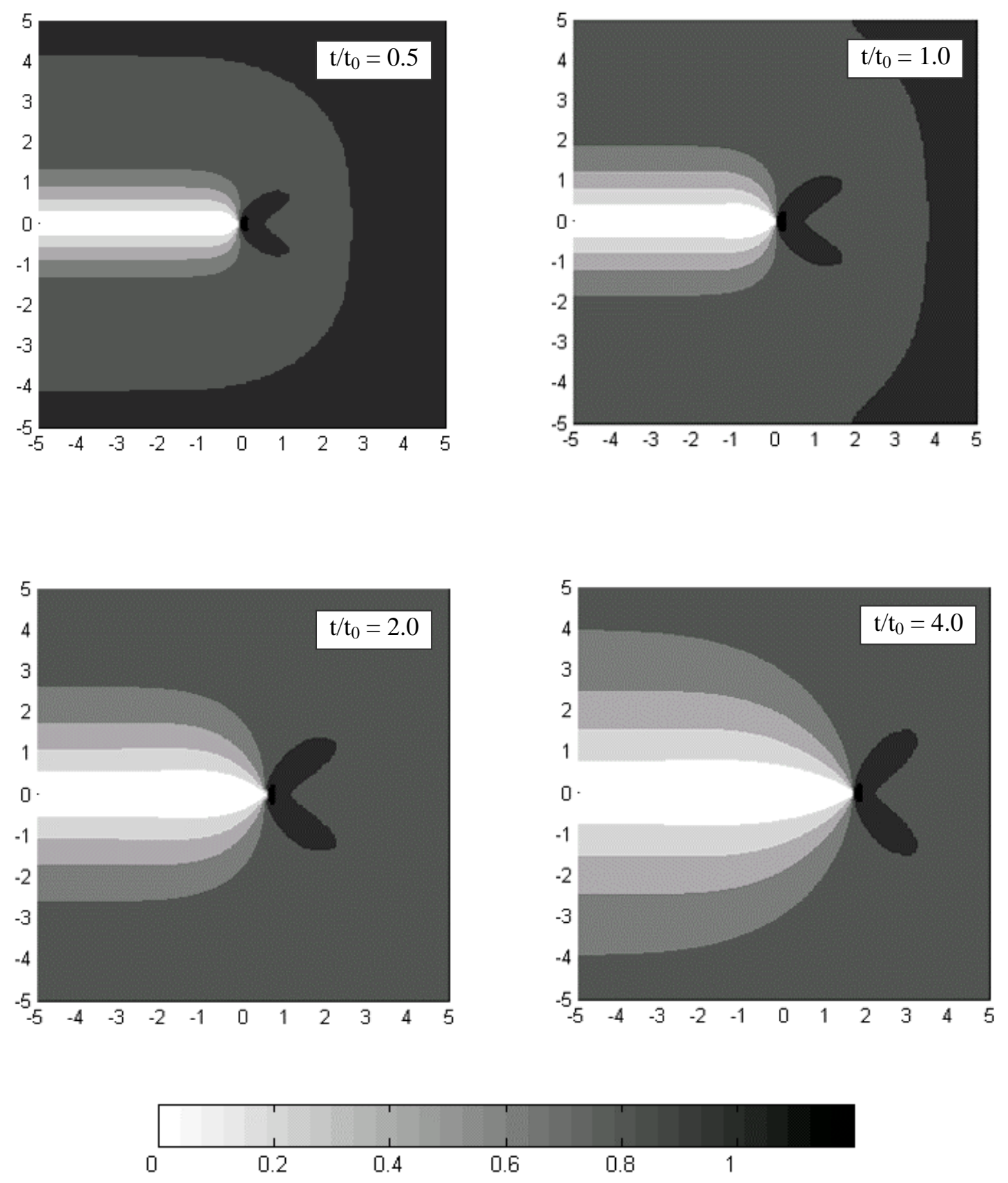

Fig. 4-The contours plots of the stress field $\sigma_{22}\left(x_{1}, x_{2}, t\right)$ in the film at several times. The coordinates $x_{1}$ and $x_{2}$ are given in the units of $\Lambda$, and the stress in the units of $\sigma_{0}$.

a robust quantity, attained by a finite crack in a finite film, so long as the lateral feature sizes (including the crack length) are large compared to the intrinsic length $\Lambda$.

\section{Equilibrium Crack in a Microbridge}

The length of the bridge introduces another dimensionless group, $L / \Lambda=L\left(\sigma_{0} / K_{c}\right)^{2}$. The width of the bridge is much larger than $\Lambda$, and so is the initial crack length. Everything else being equal, a shorter bridge stores less elastic energy, so that the crack moves slower. When the crack velocity approaches zero, the entire bridge approaches equilibrium, and the viscous stripe stops creeping and carries no stresses. In the equilibrium state, the space occupied by the stripe is equivalent to a cavity, and the bridge is in a state of plane stress with no body forces.

The stress intensity factor in this equilibrium state, $K_{\text {eq }}$, can be calculated by an established energy method. ${ }^{39}$ When a crack extends by a unit width in the bridge, the elastic energy in the sheet reduces by $h K_{\mathrm{eq}}^{2} / E .^{40}$ This energy reduction can be calculated by another method. Far behind the crack tip, the bridge is relaxed in the $x_{2}$-direction, but constrained in the $x_{1}$-direction, so that the stress state is uniaxial, $\sigma_{11}=E \varepsilon_{0}$, and the elastic energy stored in a unit width of the cracked bridge is $E \varepsilon_{0}^{2} L h$. Far ahead of the crack tip, the bridge is 


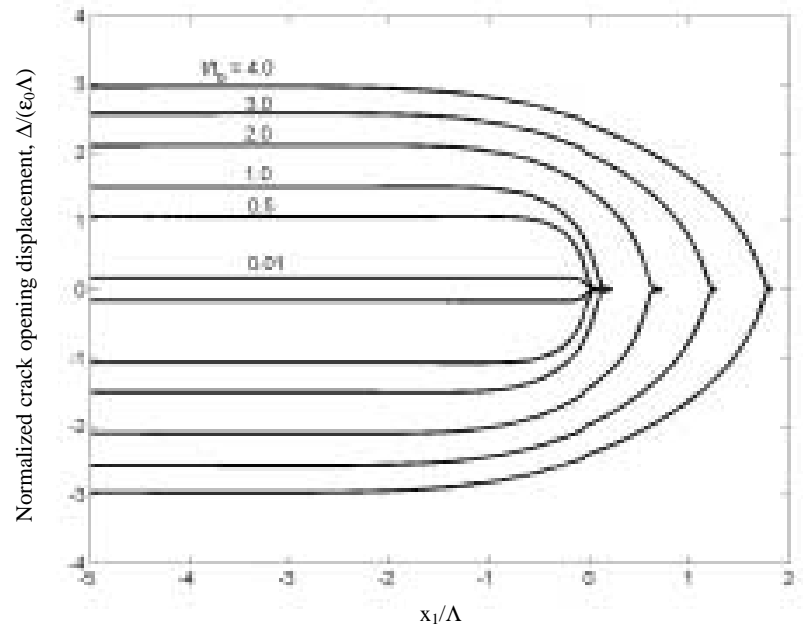

Fig. 5-The crack opening profiles at several times

in the biaxial stress state, $\sigma_{11}=\sigma_{22}=\sigma_{0}$, and the elastic energy stored in a unit width of the unrelaxed bridge is $2 E \varepsilon_{0}^{2} L h /(1-\nu)$. The extension of the crack by a unit width is equivalent to removing a unit width of the unrelaxed bridge far ahead of the crack tip, and then appending a unit width of the cracked bridge far behind of the crack tip. Equating the energy reduction calculated by the two methods, we obtain the stress intensity factor at the equilibrium state:

$$
K_{\mathrm{eq}}=\sigma_{0} \sqrt{\left(1-v^{2}\right) L} .
$$

The above argument is rigorous for a semi-infinite crack in a bridge of infinite width. Our numerical calculations show that the stress intensity factor is close to eq (17) for any crack longer than about $L$.

If $K_{\mathrm{eq}}<K_{c}$, the underlayer creep allows the film to approach equilibrium, and the crack tip will never move. Everything else being equal, a critical bridge length exists

$$
L_{c}=\frac{1}{1-\nu^{2}}\left(\frac{K_{c}}{\sigma_{0}}\right)^{2}=\frac{\Lambda}{1-\nu^{2}},
$$

below which the crack will never grow. For Poisson's ratio $\nu=0.3$, the critical bridge length is $L_{c}=1.1 \Lambda$.

Figure 6 shows the stress intensity factor for a stationary crack in the microbridge, $K$, calculated using the finite element method. For comparison, the stress intensity factor for a stationary crack in a blanket film, eq (15), is plotted as a dashed curve. Dimensional considerations dictate that $K / K_{\text {eq }}$ be a function of $t D / L^{2}$. When creep just starts, the relaxation zone in the crack wake is small compared to the bridge length $2 L$, and $K(t)$ follows the same curve as that for the blanket film. After some time $\left(t \approx L^{2} / D\right)$, the film approaches the equilibrium state, and $K / K_{\mathrm{eq}} \rightarrow 1$.

\section{Moving Crack in a Microbridge}

Now consider the case $K_{\text {eq }}>K_{c}$. As the underlayer creeps, $K$ builds up from zero to $K_{c}$. Afterwards, the crack tip moves, approaching a steady velocity. Figure 7 plots the contours of the stress $\sigma_{22}$ at time $t=3 t_{0}$ in bridges of several lengths. In each case, the crack starts at $x_{1}=0$, and has

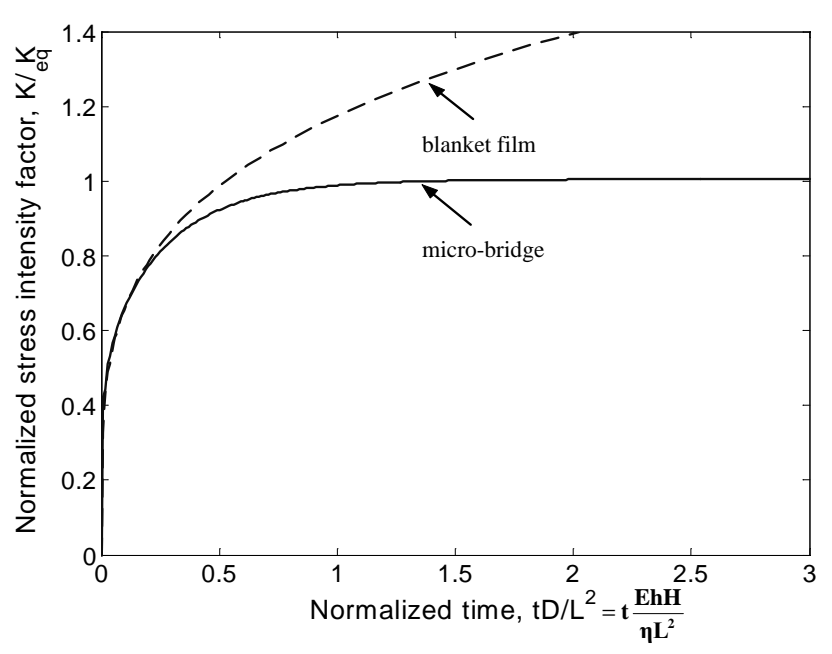

Fig. 6-The stress intensity factor of a stationary crack in a microbridge, $K$, increases with time, and approaches the equilibrium value. The stress intensity factor of a stationary crack in a blanket film is also plotted for comparison.
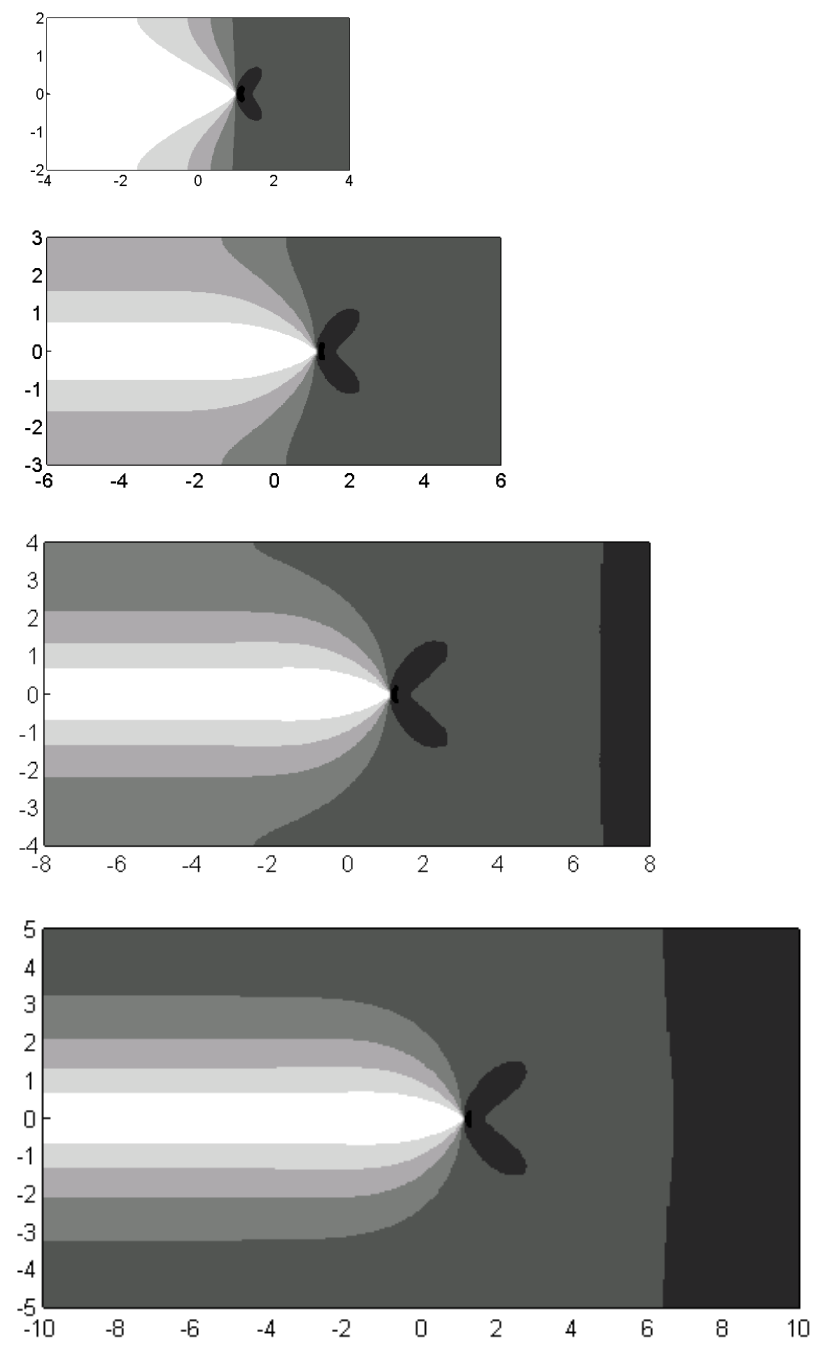

Fig. 7-The contour plots of the stress field $\sigma_{22}\left(x_{1}, x_{2}\right)$ at time $t=3 \mathrm{t}_{0}$ in microbridges of different lengths. The coordinates $x_{1}$ and $x_{2}$ are given in the units of $\Lambda$. 
attained its steady velocity by the time $t=3 t_{0}$. For a short bridge, the crack wake is relaxed across the bridge at a short distance behind the crack tip. For a long bridge, the crack wake is not fully relaxed even very far behind the crack tip.

Dimensional considerations require that the steady crack velocity in a microbridge, $V_{s S}$, take the functional form:

$$
\frac{V_{s s}}{V_{s s}^{\infty}}=f\left(\frac{K_{c}}{K_{\mathrm{eq}}}, \nu\right) .
$$

The steady crack velocity in the blanket film, $V_{s s}^{\infty}$, is given by eq (16). The equilibrium stress intensity factor, $K_{\text {eq }}$, is given by eq (17). Figure 8 shows the steady crack velocity calculated from the finite element method. When $K_{c} / K_{\text {eq }} \rightarrow$ 0 , the bridge approaches a blanket film. When $K_{c}>K_{\text {eq }}$, the crack tip will never move.

\section{Experimental Implications}

We are unaware of any systematic experimental study on concomitant thin-film cracking and underlayer creep. On the basis of the above theoretical findings, we outline experimental implications of this phenomenon. Mechanical tests using miniaturized free-standing samples have been challenging, ${ }^{41,42}$ and become impossible if the films cannot be made or handled in the free-standing form. It is urgent to develop mechanical tests that can be readily implemented in a microfabrication setting. ${ }^{43,44}$

\section{Experimental Determination of the Toughness of Brittle Films}

Ma and co-workers, of Intel Corporation, have demonstrated a technique to measure the toughness of brittle films. ${ }^{45}$ On a silicon substrate, a metal layer was deposited, on top of which a brittle film was deposited. The structure looked like that in Fig. 1(a), with the viscous layer replaced by the metal layer. In the time duration of the experiment, the metal layer did not creep, but was plastically deformable. The film was under residual tensile stress. Large crack-like flaws were introduced by scratching the film. The residual stress by itself, however, was insufficient to drive the scratch flaws to grow into channel cracks. The whole structure was then subject to a bending moment, until the scratch flaws grew into channel cracks, running in the direction normal to the bending stress. The net stress in the film is the sum of the residual stress and the bending stress. The critical stress was recorded, and related to the toughness of the film.

This technique uses the established microfabrication processes and mechanical testing procedures. It determines the critical stress accurately by controlling the bending moment and observing the crack growth in an optical microscope in situ. The technique exploits a specific result of the thin film fracture mechanics. After the crack grows a small distance away from the scratched zone, the stress intensity factor at the crack tip is independent of the scratch geometry, and is simply proportional to the net stress in the film and the square root of the film thickness. ${ }^{16-19}$ Consequently, no particular care is needed in introducing the flaws.

Two prerequisites limit the applications of this technique: the film must be sufficiently stiff and have appreciable tensile residual stress. For a compliant film on a stiff substrate, the bending moment generates a smaller stress in the film than in the substrate, and may break the substrate. Similarly, if the residual stress in the film is small, a large bending moment is needed, which may also break the substrate.

To avoid these problems, we suggest that a microbridge is used; see Figs. 1(b) or 1(c). Under the bridge lies a viscous stripe, or just a cavity if the stripe is removed in fabrication. We can view the bridge as a tensile specimen, with the gage length $2 L$ and the two ends gripped by the substrate. Following Ma et al., ${ }^{45}$ we can introduce flaws by scratching the film. We then determines the critical stress that drives the flaws to grow into channel cracks by controlling the bending moment and observing the crack growth in a microscope. The equilibrium stress intensity factor is given by eq (17), which is now proportional to the square root of the bridge length, rather than its thickness. Even a small stress will drive a channel crack in a long bridge. If a viscous stripe lies under the bridge, we should control the bending moment to drive the crack at a sufficiently low velocity; the time needed for the bridge to attain the equilibrium state scales as $L^{2} / D .^{27-29}$ This time requirement is removed if the bridge is over a cavity.

\section{Experimental Determination of Creep Laws}

If the properties of the elastic film are known, the measured crack velocity can be used to determine the creep properties of the underlayer. For example, when the underlayer in Fig. 1(a) is linear viscous, once the velocity for a crack channeling in a blanket film is measured experimentally, the viscosity $\eta$ is determined by eq (16). The total distance traversed by the crack is the sample size, and can be readily observed in a microscope. By contrast, the total displacement scales with the sample size times the average strain, and is small for a small specimen. In principle, the proposed method can measure viscosity of arbitrarily thin layers.

If the underlayer has a more complex creep behavior, additional calculations are needed to interpret the experiment. We will pursue this matter in detail in a separate study. Here we give the scaling law of the channel crack velocity, assuming that the underlayer creeps according to the power law. That is, the shear strain-rate $\dot{\gamma}$ relates to the shear stress $\tau$ according to

$$
\dot{\gamma}=B \tau^{n}
$$

where $B$ and $n$ are parameters to fit experimental data. This creep law now replaces the linear viscous law (4). Following the same line of reasoning that leads to eq (16), we obtain the scaling law for the crack velocity channeling in the elastic film:

$$
V_{s s}^{\infty}=\chi E B H \sigma_{0}^{n-1} h^{n}\left(\sigma_{0} / K_{c}\right)^{2 n}
$$

The dimensionless factor $\chi$ now is a function of Poisson's ratio and creep exponent, $\chi(v, n)$, to be calculated by using the finite element method in the subsequent work. We can vary the stress in the elastic film by the bending moment, and measure the crack velocity as a function of the stress. Such experimental data would be sufficient to determine the creep properties $B$ and $n$.

\section{Subcritical Decohesion}

In the above discussion, we have assumed that the crack grows when the stress intensity factor at the crack tip equals the toughness, $K=K_{c}$. In reality, many brittle materials suffer subcritical decohesion, in which the crack tip moves 


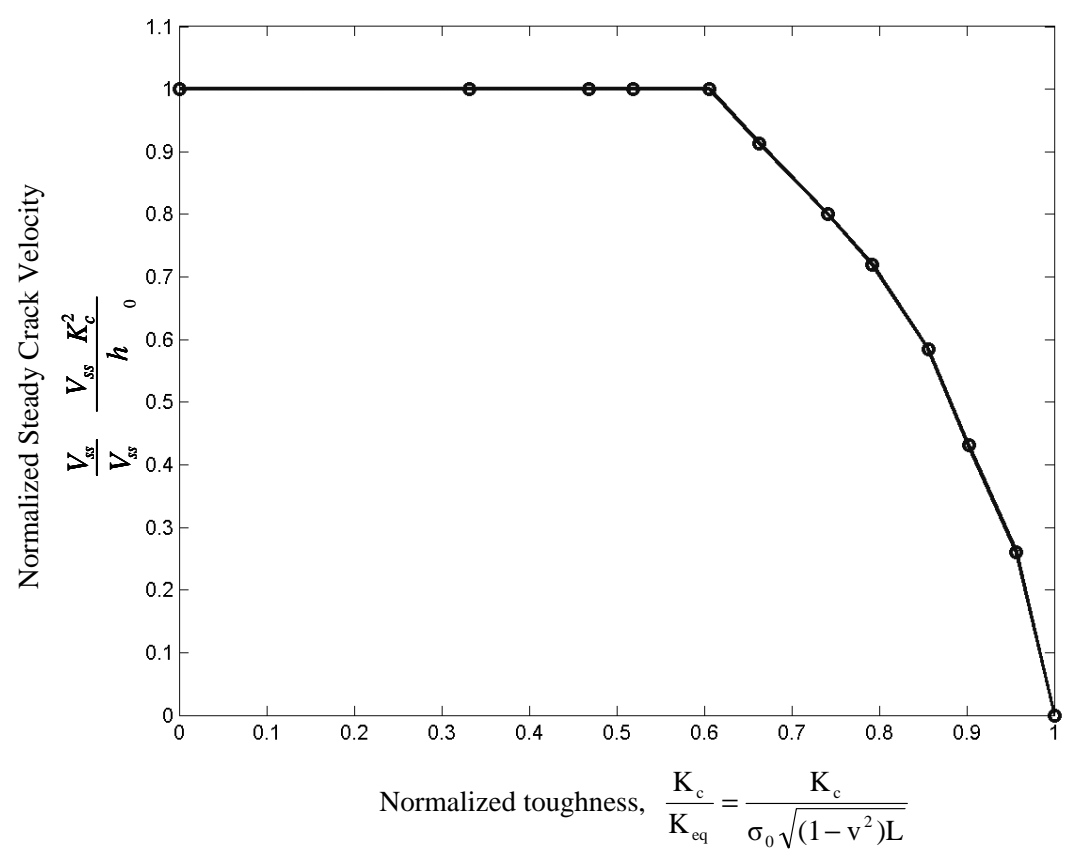

Fig. 8-The normalized steady crack velocity in the microbridge as a function of the normalized toughness

at a small velocity even when $K<K_{c}{ }^{46}$ Molecules, such as $\mathrm{H}_{2} \mathrm{O}$, in the environment diffuse to the crack tip and assist in breaking the atomic bonds. Figure 9 sketches the trend of the representative experimental data. The crack tip is stationary when $K$ is below a threshold value, $K_{t h}$, and moves when $K>K_{t h}$. The crack velocity $V$ increases with the stress intensity factor $K$. When the crack tip moves so fast that the environmental molecules cannot assist in breaking atomic bonds at the crack tip, $K=K_{c}$.

Now consider a crack in a film of such a material on a creeping underlayer. Before creep starts, the stress in the film is uniform, so that $K=0$. When the underlayer creeps, $K$ increases with time, and the crack tip remains stationary until $K=K_{t h}$. When the crack tip starts to move, the stress intensity factor at the crack tip, as well as the crack velocity, is determined by the two rate processes: the subcritical decohesion in the film, and the creep in the underlayer. After some transient motion, the crack tip may attain a steady velocity $V_{s s}$ and a steady stress intensity factor $K_{s s}$. All previous results on the steady crack velocity are applicable, provided $K_{c}$ is replaced by $K_{s s}$. For example, we can rewrite eq (19) as

$$
V_{s s}=\chi \frac{H h E \sigma_{0}^{2}}{\eta K_{s s}^{2}} f\left(\frac{K_{s s}}{\sigma_{0} \sqrt{\left(1-v^{2}\right) L}}, \nu\right)
$$

Here we have used eq (16) for $V_{s s}^{\infty}$, and eq (17) for $K_{\text {eq }}$. Also sketched in Fig. 9 is the $V_{s s}-K_{s s}$ relation (22), which can be varied by varying the stress $\sigma_{0}$ in the film and the length $2 L$ of the microbridge, among other quantities. The intersection of the two curves selects both the steady state stress intensity factor $K_{s s}$ and the crack velocity $V_{s s}$.

The channel crack cannot grow in the bridge if $K_{\text {eq }}<K_{t h}$. That is, the crack can equilibrate if the bridge length is below a threshold, given by

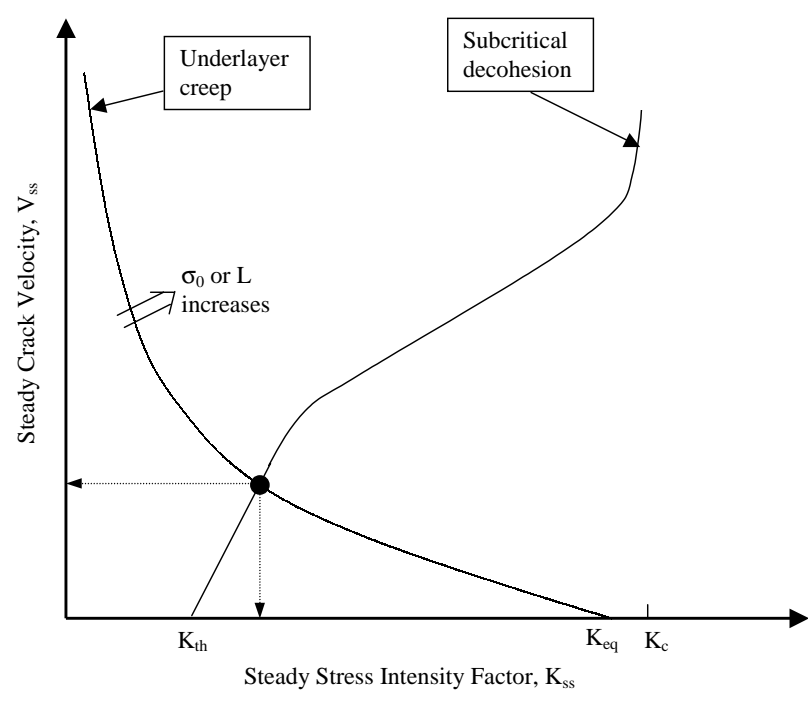

Fig. 9-The crack velocity as a function of the stress intensity factor. One curve is the $V--K$ relation determined by underlayer creep, and the other is the $V--K$ relation determined by subcritical decohesion. The intersection of the two curves selects the steady-state crack velocity and stress intensity factor.

$$
L_{t h}=\frac{1}{1-v^{2}}\left(\frac{K_{t h}}{\sigma_{0}}\right)^{2} .
$$

This threshold replaces eq (18). We can fabricate an array of microbridges of different lengths on the same wafer, scratch them all, and bend the wafer with a constant moment. After some time, channel cracks form in long bridges, but not in 
short ones. Experiments of this kind may be used to determine $K_{t h}$, or even the entire subcritical decohesion $K-V$ curve.

\section{Ratcheting-induced Crack Growth}

As mentioned in the introduction, an unexpected finding made recently in thin-film mechanics is that cracks can grow in brittle films under cyclic temperatures, driven by ratcheting plastic deformation in a metal underlayer. ${ }^{11-15}$ This phenomenon may be contrasted with fatigue cracks commonly observed in ductile materials subject to cyclic loads. ${ }^{47}$ A previous study calculated the ratcheting displacement in the crack wake, but did not obtain the growth rate of the crack. ${ }^{13}$ We now derive a scaling law for the crack growth per temperature cycle.

We replace the viscous layer in Fig. 1(a) by a metal layer, and cycle the structure between temperatures $T_{L}$ and $T_{H}$. Within the temperature and time of the experiment, the metal does not creep, but can have time-independent plastic deformation. For simplicity, we assume that the tensile stress in the uncracked blanket film, $\sigma_{0}$, is constant as the temperature changes. This occurs in practice when the elastic film and the substrate have a similar coefficient of thermal expansion, and $\sigma_{0}$ is due to the deposition process or the bending moment.

First, we consider a blanket metal film on a substrate, subject to a constant shear stress $\tau$ on the film surface and the cyclic temperatures. The temperature range $T_{H}-T_{L}$ is large enough so that the difference in the thermal expansion coefficients between the metal and the substrate causes the metal to yield. Each cycle, the metal film deforms plastically in the direction of the shear stress $\tau$. When the temperature range is large and the shear stress is small, the plastic shear strain per cycle, $\mathrm{d} \gamma^{p} / \mathrm{d} N$, is linear in the shear stress, namely ${ }^{12}$

$$
\frac{\mathrm{d} \gamma^{p}}{\mathrm{~d} N}=\frac{\tau}{\eta_{R}}
$$

where

$$
\eta_{R}=\frac{E_{m}}{12\left(1-v_{m}\right)}\left[\frac{E_{m}\left(\alpha_{m}-\alpha_{s}\right)\left(T_{H}-T_{L}\right)}{\left(1-v_{m}\right) Y}-2\right]^{-1}
$$

Here $E_{m}$ is Young's modulus, $\nu_{m}$ is Poisson's ratio, and $Y$ is the uniaxial yield strength of the metal. $\alpha_{m}$ and $\alpha_{s}$ are the coefficients of thermal expansion of the metal and substrate. Equation (24) is analogous to the linear viscous law (4) in that the number of cycles $N$ is analogous to the time $t$, and the parameter $\eta_{R}$ is analogous to the viscosity $\eta$.

Now we put an elastic film on top of the metal layer. As temperature cycles, the metal layer undergoes ratcheting plastic deformation. The stress field in the film relaxes in the crack wake, and intensifies around the crack tip. The behavior should be the same as shown in Figs. 3-5, if we replace the time by the number of cycles. By the ratcheting-creep analogy, we rewrite the scaling law for the crack velocity (16) as the scaling law for crack growth per cycle:

$$
\frac{\mathrm{d} a}{\mathrm{~d} N}=\chi \frac{H h E \sigma_{0}^{2}}{\eta_{R} K_{c}^{2}}
$$

The pre-factor $\chi$ takes the same value as before; for example, $\chi=0.534$ when Poisson's ratio is 0.3 for the film. We can similarly obtain the growth rate for a crack in an elastic bridge over a metal stripe. Experimental observations of ratchetinginduced crack growth have been reported for interconnect structures, ${ }^{11,12}$ and for oxide scales on alloys. ${ }^{9,10}$ Quantitative measurement of the crack growth rate is unavailable.

\section{Concluding Remarks}

In this paper we study crack extension in a brittle film concomitant with creep in an underlayer. We use a twodimensional shear lag model to approximate the threedimensional fracture process, and the extended finite element method to simulate the moving crack with an invariant, relatively coarse mesh. The crack tip may remain stationary, and the film approaches an equilibrium state. Alternatively, the crack tip may move and quickly approaches a steady velocity. Both the equilibrium state and the steady motion can be realized under a wide range of conditions. We suggest novel methods to measure toughness and inelastic properties in thin-film structures. Using the ratcheting-creep analogy, we obtain the growth per temperature cycle of a channel crack in an elastic film over a metallic layer. It is hoped that experiments will soon succeed in exploiting these ideas.

After reading the preprint of this paper, Professor James R. Rice, of Harvard University, kindly told us the history of the shear lag model in geophysics, where the lithosphere was modeled as an elastic layer, and the asthenosphere as a viscous underlayer. In particular, his group studied the steady crack growth under a shear stress in an elastic layer on a viscoelastic underlayer. $^{48}$

\section{Acknowledgments}

This work is supported partly by the National Science Foundation through grants CMS-9820713 and CMS9988788 with Drs Ken Chong and Jorn Larsen-Basse as Program Directors, and partly by the New Jersey Science and Technology Commission. Discussions with Drs Jun He and Qing Ma, of Intel Corporation, have been helpful.

\section{References}

1. M.D. Thouless, “Cracking and Delamination of Coatings,” J. Vac. Sci. Technol. A 9, 2510-2515 (1991).

2. Hutchinson, J.W. and Suo, Z., "Mixed-mode Cracking in Layered Materials," Advances in Applied Mechanics, 29, 63-191 (1992).

3. Evans A.G. and Hutchinson J.W., "The Thermomechanical Integrity of Thin Films and Multilayers,” Acta Metall. Mater., 43, 2507-2530 (1995).

4. Cook, R.F. and Suo, Z., "Mechanisms Active during Fracture under Constraint," MRS Bulletin, 27, 45-51 (2002).

5. Suo, Z., "Reliability of Interconnect Structures." A Manuscript prepared as a chapter in Volume 8. Interfacial and Nanoscale Failure, W. Gerberich and W. Yang, eds., Comprehensive Structural Integrity, I. Milne, R.O. Ritchie, and B. Karihaloo, editors-in-Chief. Due for publication early 2003. (Preprint available online at http://www.princeton.edu/ suo, Publication 139.)

6. Kook, S.-Y. and Dauskardt, R.H., "Moisture-assisted Subcritical Debonding of a Polymer/Metal Interface,” J. Appl. Phys., 91, 1293-1303 (2002).

7. Soboyejo, W.O., Wang, R., Katsube, N., Seghi, R., Pagedas, C., Skraba, P., Mumm, D.R., and Evans, A.G., "Contact Damage of Model Dental Multilayers: Experiments and Finite Element Simulations,” Key Engineering Materials, 198-199, 135-178 (2001).

8. Kahn, H., Heuer, A.H., and Ballarini, R., "On-chip Testing of Mechanical Properties of MEMS Devices,” MRS Bulletin, 26, 300-301 (2001).

9. Muhlstein, C.L., Stach, E.A., and Ritchie, R.O., "Mechanism of Fatigue in Micron-scale Films of Polycrystalline Silicon for Microelectromechanical Systems,” Appl. Phys. Lett., 80 1532-1534 (2002).

10. Evans, A.G., Mumm, D.R., Hutchinson, J.W., Meier, G.H., and Pettit, F.S., "Mechanisms Controlling the Durability of Thermal Barrier Coatings," Progress in Materials Science, 46, 505-553 (2001). 
11. Huang, M., Suo, Z., Ma, Q., and Fujimoto H., "Thin Film Cracking and Ratcheting Caused by Temperature Cycling,” J. Mater. Res., 15, 1239-1242 (2000).

12. Huang, M., Suo, Z., and Ma, Q., "Plastic Ratcheting Induced Cracks in Thin Film Structures,” J. Mech. Phys. Solids, 50, 1079-1098 (2002).

13. Begley, M.R. and Evans, A.G., "Progressive Cracking of a Multilayer System upon Thermal Cycling,” J. Appl. Mech., 68, 513-520 (2001).

14. He, M.Y., Evans, A.G., and Hutchinson, J.W., "The Ratcheting of Compressed Thermally Grown Thin Films on Ductile Substrates," Acta Mater., 48, 2593-2601 (2000).

15. Karlsson, A.M. and Evans, A.G., "A Numerical Model for the Cyclic Instability of Thermally Grown Oxides in Thermal Barrier Systems," Acta Mater., 49, 1793-1804 (2001).

16. Beuth, J.L., "Cracking of Thin Bonded Films in Residual Tension," Int. J. Solids Structures, 29, 1657-1675 (1992).

17. Ye,T., Suo, Z., and Evans, A.G., "Thin Film Cracking and the Roles of Substrate and Interface," Int. J. Solids Structures, 29, 2639-2648 (1992).

18. Hu, M.S. and Evans, A.G., "The Cracking and Decohesion of Thin Films on Ductile Substrates,” Acta Metall., 37, 917-925 (1989).

19. Beuth, J.L. and Klingbeil, N.W., "Cracking of Thin Films Bonded to Elastic-plastic Substrates,” J. Mech. Phys. Solids, 44, 1411-1428 (1996).

20. Leterrier, Y., Boogh, L., Andersons, J. and Mansons, J-A. E., “Adhesion of Silicon Oxide Layers on Poly(ethylene-terephthalate) I: Effect of Substrate Properties on Coatings Fragmentation Process," J. Polym. Sci. Part B. Polm. Phys. 35, 1449-1461 (1997).

21. Hobart, K.D., Kub, F.J., Fatemi, M., Twigg, M.E., Thompson, P.E., Kuan, T.S., and Inoki, C.K., "Compliant Substrates: A Comprehensive Study of the Relaxation Mechanisms of Strained Films Bonded to High and Low Viscosity Oxides,” J. Electron. Mater., 29, 897-900 (2000).

22. Yin, H., Huang, R., Hobart, K.D., Suo, Z., Kuan, T.S., Inoki, C.K., Shieh, S.R., Duffy, T.S., Kub, F.J., and Sturm, J.C., "Strain Relaxation of SiGe Islands on Compliant Oxide,” J. Appl. Phys., 91, 9716-9722 (2002).

23. Wagner, H.S., Gleskova, H., Sturm, J.C., and Suo, Z., "Novel Processing Technology for Macroelectronics," in Technology and Applications of Amorphous Silicon, R.A. Street, ed., pp. 222-251, Springer, Berlin (2000).

24. Sikder, A.K., Irfan, I.M., Kumar, A., and Anthony J.M., "NanoIndentation Studies of Xerogel and Silk Low-k Dielectric Materials,” J. Electron. Mater., 30, 1527-1531 (2001).

25. Toivola, Y., Thurn, J., Cook, R.F., "Structural, Electrical, and Mechanical Properties Development During Curing Of Low-k Hydrogen Silsesquioxane Films,” J. Electrochem. Soc. 149, F9-F17 (2002).

26. Huang, R., Prévost, J.H., and Suo, Z., "Loss of Constraint on Fracture in Thin Film Structures Due to Creep," Acta Mater., 50, 4137-4148 (2002).

27. Freund, L.B. and Nix, W.D., unpublished work.

28. Moran, P.D. and Kuech, T.F., "Kinetics of Strain Relaxation in Semiconductor Films Grown on Borosilicate Glass-bonded Substrates,” J. Electron. Mater., 30, 802-806 (2001).

29. Huang, R., Yin, H., Liang, J., Hobart, K.D., Sturm, J.C., and Suo, Z., "Relaxation of a Strained Elastic Island on a Viscous Layer," Mater. Res. Soc. Symp. Proc., 695, 115-120 (2001).
30. Dmowska, R. and Rice, J.R., "Fracture Theory and Its Seismological Applications," in Continuum Theories in Solid Earth Physics, R. Teisseyre, ed., Physics and Evolution of the Earth's Interior, Vol. 3, pp.187-255, Elsevier, Oxford (1986).

31. Freund, L.B., Dynamic Fracture Mechanics, Cambridge University Press (1990).

32. Nix, W.D. “Mechanical Properties of Thin Films,” Metall. Trans. A, 20, 2217-2245 (1989).

33. Baker, S.P., Kretschmann, A., and Arzt, E., "Thermomechanical Behavior of Different Texture Components in Cu Thin Films," Acta Mater., 49, 2145-2160 (2001).

34. Belytschko, T. and Black, T., "Elastic Crack Growth in Finite Elements with Minimal Remeshing,” Int. J. Numer. Methods Eng., 45, 601-620 (1999).

35. Moës, N., Dolbow, J., and Belytschko, T., “A Finite Element Method for Crack Growth without Remeshing,” Int. J. Numer. Methods Eng., 46, 131-150 (1999).

36. Daux, C., Moës, N., Dolbow, J., Sukumar, N., and Belytschko, T., “Arbitrary Branched and Intersecting Cracks with the Extended Finite Element Method,” Int. J. Numer. Methods Eng., 48, 1741-1760 (2000).

37. Sukumar, N., Srolovitz, D.J., Baker, T.J., and Prévost, J.H., "Brittle Fracture in Polycrystalline Microstructures with the Extended Finite Element Method," Int. J. Numer. Methods Eng., in press.

38. Prévost, J.H., "DYNAFLOW: A Nonlinear Transient Finite Element Analysis Program,” Princeton University (1981). Last updated in 2002.

39. Rice, J.R., "A Path Independent Integral and Approximate Analysis of Strain Concentration by Notches and Cracks,” J. Appl. Mech., 35, 379-386 (1968).

40. Irwin, G. R., "Analysis of Stresses and Strains Near the End of a Crack Traversing a Plate,” J. Appl. Mech. 24, 361-364 (1957).

41. Huang, H.B. and Spaepen, F., "Tensile Testing of Free-standing Cu, Ag and Al Thin Films and Ag/Cu Multilayers,” Acta Mater. 48, 3261-3269 (2000).

42. LaVan D.A., Sharpe W.N., "Tensile Testing of Microsamples," EXPERIMENTAL MECHANICS, 39, 210-216 (1999).

43. Kraft, O. and Volkert, C.A., "Mechanical Testing of Thin Films and Small Structures,” Adv. Eng. Mater., 3 (3), 99-110 (2001).

44. Vinci, R.P., Baker, S.P., eds., "Mechanical Properties in Small Dimensions," MRS Bulletin, 27, 12-53 (2002).

45. Ma, Q., Xie, J., Chao, S., El-Mansy, S., McFadden, R., and Fujimoto, H., "Channel Cracking Technique for Toughness Measurement of Brittle Dielectric Thin Films on Silicon Substrates,” Mater. Res. Soc. Symp. Proc. 516, 331-336 (1998).

46. Lawn, B., Fracture of Brittle Solids, 2nd edition, Cambridge University Press (1993).

47. Suresh, S., Fatigue of Materials, 2nd edition, Cambridge University Press (1998).

48. Lehner, F.K., Li, V.C., and Rice, J.R., "Stress Diffusion Along Rupture Plate Boundaries," Journal of Geophysical Research, 86, 6155-6169 (1981). 